\title{
Controversies in perioperative troponin elevation in non-cardiac surgery
}

\author{
Nirvik Pal, Eric Worrall, Aaron Lim \\ Department of Anesthesiology, Virginia Commonwealth University, Richmond, VA 23298, USA.
}

Correspondence to: Associate Prof. Nirvik Pal, Department of Anesthesiology, PO Box 980541, 1250 East Marshall St, 5th Floor, Virginia Commonwealth University, Richmond, VA 23298, USA. E-mail: nirvik.pal@vcuhealth.org

How to cite this article: Pal N, Worrall E, Lim A. Controversies in perioperative troponin elevation in non-cardiac surgery. Vessel Plus 2021;5:20. https://dx.doi.org/10.20517/2574-1209.2020.57

Received: 16 Oct 2020 First Decision: 10 Nov 2020 Revised: 16 Nov 2020 Accepted: 25 Jan 2021 Available online: 23 Apr 2021

Academic Editors: Deborah B. Diercks, Keith A. Webster Copy Editor: Xi-Jun Chen Production Editor: Yue-Yue Zhang

\begin{abstract}
The Fourth Universal Definition of Myocardial Infarction recognizes "myocardial injury" as a distinct entity with a milder form of myocardial necrosis leading to the release of detectable troponin. Myocardial injury after noncardiac surgery (MINS) is the release of detectable troponin in the absence of symptoms within 30-day. MINS has been increasingly recognized as it has a prognostic bearing on outcomes. However, the diagnosis, interpretation, implication, and treatment strategies are yet to be resolved. Definitions of diagnosis have changed from the fourth generation to fifth-generation (high-sensitivity troponin), which still is proprietary and lack universal standardization; interpretation in studies have varied depending on definitions based on fourth or fifth generation troponin assays. Other than prognostic implications, either equivocal results or limited studies exist for the utility for prevention and screening of MINS. Additionally, the studied strategies and drugs to prevent MINS have either failed to translate in clinical trials in humans, or showed inconclusive results. We, as a result of this, in our review, highlight such facts regarding elevation in perioperative troponin faced by the practicing perioperative clinician regularly.
\end{abstract}

Keywords: Perioperative myocardial infarction, myocardial injury, myocardial injury after non-cardiac surgery, high-sensitivity troponin, cardiac troponin

\section{INTRODUCTION}

The number of surgeries performed annually worldwide exceeds 300 million and is ever-growing ${ }^{[1,2]}$. Each year more than 10 million patients develop major cardiac complications in the perioperative period ${ }^{[3]}$. In the United States, the prevalence of major adverse cardiac and cerebrovascular events is 1 in 33 hospitalizations 
for non-cardiac surgery ${ }^{[4]}$. Most studies looking at perioperative cardiac complications have focused on myocardial infarction (MI), defined as myocardial necrosis due to myocardial ischemia and clinically diagnosed by elevated troponins in the setting of ischemic symptoms or electrocardiogram (ECG) finding $\mathrm{s}^{[5]}$. However, in the perioperative setting, many patients sustain myocardial injury without meeting the criteria for MI. To better identify and define this population, which may portend a poor prognosis and warrant intervention, the term "myocardial injury after non-cardiac surgery (MINS)" was created. MINS is prognostically relevant myocardial injury due to ischemia that occurs during or within 30 days after noncardiac surgery and is diagnosed by elevated troponins but does not require the presence of symptoms $\mathrm{s}^{[5]}$ ". In 2018, the Fourth Universal Definition of Myocardial Infarction defined "myocardial injury" as cardiac biomarker release without evidence of myocardial ischemia - such as ischemic symptoms, new ECG changes or regional wall motion abnormalities, loss of myocardial viability, or intracoronary thrombus ${ }^{[6]}$. These two definitions may create confusion as to the former attributes myocardial injury to ischemia, while the latter does not, leaving clinicians to wonder whether myocardial injury and MINS are competing or overlapping diagnoses. Furthermore, clinicians may incorrectly diagnose a real acute coronary syndrome (ACS) - which may mandate immediate intervention like coronary revascularization - as MINS or myocardial injury ${ }^{[7]}$. Finally, it is unclear whether MINS may be genuinely a precursor to acute myocardial infarction (AMI).

\section{THE KNOWN KNOWN}

Perioperative myocardial ischemia is common and impacts patient outcomes ${ }^{[5,8]}$. Isolated troponin elevation after surgery is associated with a two-fold increase in 1-year mortality ${ }^{[9]}$ [Figure 1]. Over time, improved diagnostic accuracy and sensitivity of cardiac enzyme assays have led to fewer diagnoses of "unstable angina" and more diagnoses of non-ST-elevation myocardial infarction (NSTEMI ${ }^{[10]}$. Improved enzyme assays used in surgical patients have driven the evolution of MINS $^{[11]}$. Unfortunately, with only limited or non-conclusive trials, we have a limited consensus regarding ideal therapy or management of perioperative $\mathrm{MINS}^{[12]}$.

\section{Pathophysiology}

Both cTn-I and cTn-T are proteins within a troponin complex of thin filaments that form the contractile apparatus, or sarcomere, in cardiac myocytes ${ }^{[13]}$. Troponin is released following myocardial stress by one of three mechanisms - reversible injury, programmed cell death (apoptosis), or irreversible myocyte necrosis leading to possible clinical systemic cTn increase, detectable systemic cTn increase, or systemic cTn release proportional to the extent of necrosis, respectively ${ }^{[13,14]}$. At the molecular level, structural proteins like cTn are released systemically in the presence of a "leaky" plasma membrane and the dissociation of cellular structures like contractile filaments. Experimentally, necrosis of at least $40 \mathrm{mg}(\sim 0.015 \%)$ of the myocardium is required to systemically detect $\mathrm{cTn}>$ 99th percentile of the upper reference limit ${ }^{[15]}$. $\mathrm{cTn}$ is catabolized by the liver, pancreas, and kidneys or as well as by receptor-mediated endocytosis to the endoplasmic reticulum. Impaired clearance may lead to prolonged cTn biomarker detection. Initially, the half-life of both $c \operatorname{TnT}$ and $\mathrm{cTnI}$ is $30 \mathrm{~min}$ (in dogs and rats). Thereafter, there is a rise in cTnT owing to a more significant relative decline in clearance being more-renal dependent ${ }^{[14]}$.

\section{Myocardial ischemia may result from various mechanisms ${ }^{[16]}$}

\section{Plaque rupture and thrombosis}

"Vulnerable plaque" is a coronary plaque with a fibrous cap and necrotic lipid core involving $>40 \%$ of the lesion. When these plaques rupture, the result may be thrombosis and complete coronary arterial occlusion resulting in ST-elevation myocardial infarction (STEMI). Plaque rupture can be precipitated by 


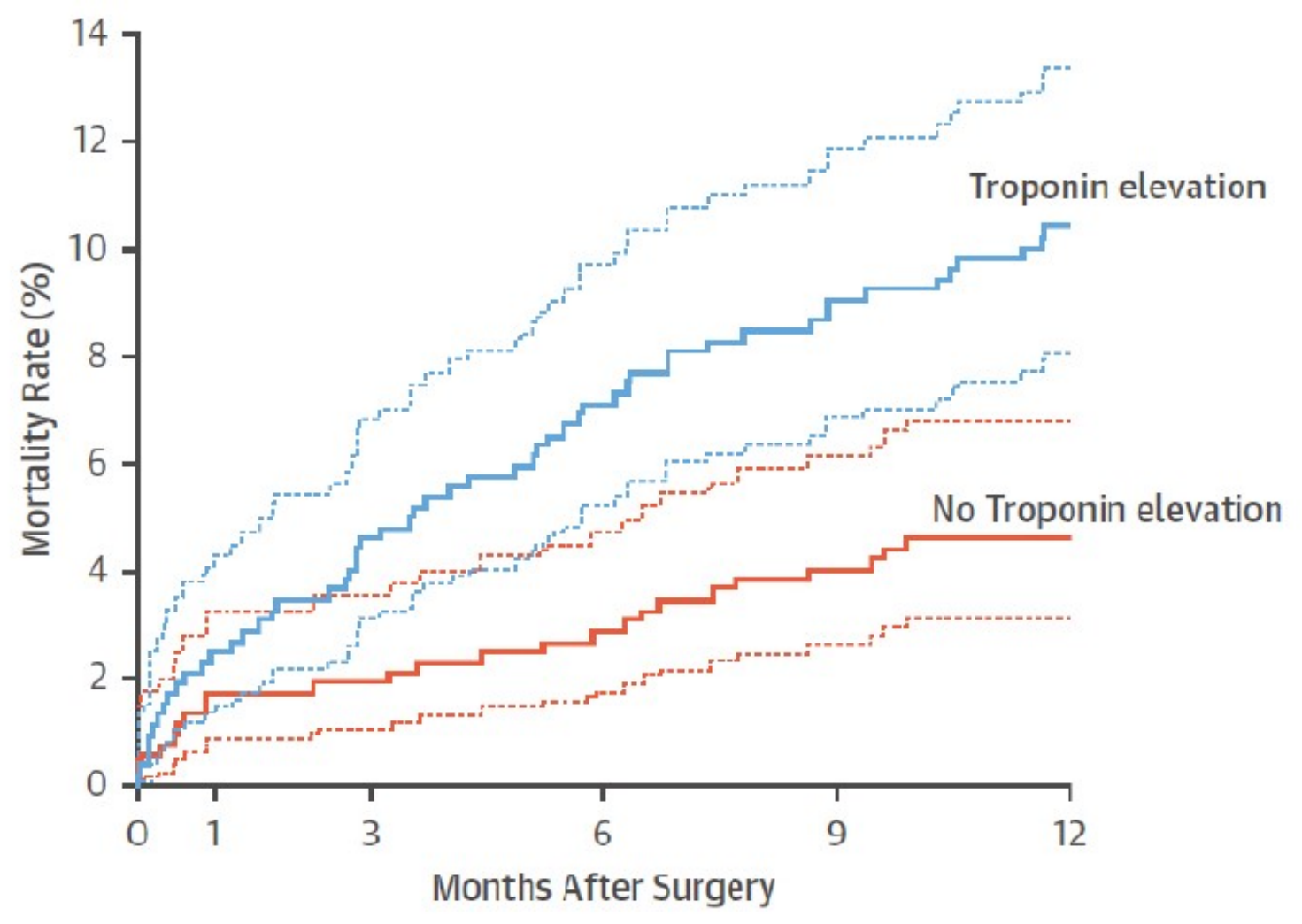

Blue lines indicate mortality in patients with a post-operative finding of isolated myocardial injury during the 3 days after surgery. Orange lines indicate patients without troponin elevation. Dotted lines indicate the upper and lower 95\% confidence limits.

Figure 1. One-year mortality in the propensity - matched cohort of 1,042 patients without adverse post-operative event ${ }^{[9]}$.

hemodynamic perturbations, turbulent flow, platelet aggregation, and coronary spasm.

Oxygen demand vs. supply imbalance

Typically, there is a linear relationship between coronary blood supply and myocardial oxygen consumption. Left ventricular perfusion occurs during diastole only and therefore is dependent on diastolic blood pressure and time (greater perfusion with lower heart rate). In contrast, right ventricular perfusion occurs in both systole and diastole.

Coronary perfusion pressure $(\mathrm{CPP})=$ Diastolic blood pressure $(\mathrm{DBP})$ - Left ventricular end-diastolic pressure (LVEDP)

\section{Critical stable stenosis}

Preserved autoregulation in the presence of reduced flow due to stenosis (stable plaque) upstream leads to vasodilation downstream. With critical stenosis, no additional hyperemic response may be possible. This has been observed with lesions occupying > 80\% diameter. 


\section{Coronary artery spasm}

Hypocapnia and intracellular calcium concentration have been associated with this phenomenon. Perioperative coronary vasospasm has been observed in patients with a history of Prinzmetal angina (coronary artery spasm).

\section{Perioperative cardiac risk evaluation}

\section{Clinical risk indices}

Several perioperative cardiac risk prediction indices ${ }^{[17]}$. While larger studies such as NSQIP generally have greater predictive accuracy, smaller, more focused studies are usually more clinically applicable ${ }^{[3]}$.

\section{Non-invasive testing}

Studies on preoperative non-invasive testing have been inconclusive. While patients with evidence of ischemia on myocardial perfusion imaging have shown to be at increased risk of perioperative cardiac complications, a meta-analysis showed one-third of myocardial infarctions or deaths occurred in patients with normal results on thallium stress testing ${ }^{[3]}$. In a multicenter prospective cohort study, preoperative testing with coronary computed tomographic angiography improved the estimation of risk for MI or death compared to the use of RCRI alone but also overestimated the risk in patients who did not have an adverse event.

\section{Measuring cardiac biomarkers [Figure 2]}

BNP and NT-proBNP: BNP (B-type natriuretic peptide) > $92 \mathrm{ng} / \mathrm{L}$ or NT-proBNP (N-terminal-pro hormone BNP) > $300 \mathrm{ng} / \mathrm{L}$ has been associated with MI within 30 days of noncardiac surgery (HR: 3.40; $95 \%$ CI: $2.57-4.47)^{[18]}$.

Troponin: MINS has been defined previously based on fourth-generation Troponin T assay, and after that currently based on fifth-generation high-sensitivity troponin (hs-cTn).

The fourth-generation troponin $\mathrm{T}>0.03 \mathrm{ng} / \mathrm{mL}$ is the threshold to define MINS and was associated with 30-day mortality (HR: 3.9; 95\%CI: 3.0-5.1 ${ }^{[5,19]}$. Whereas, the fifth generation hs-cTn, any value of > 99th percentile is considered abnormal and classified as "troponin elevation". Diagnosis of MINS requires a postoperative hs-TnT of $20 \mathrm{ng} / \mathrm{L}$ to $65 \mathrm{ng} / \mathrm{L}$ with an absolute increase of at least $5 \mathrm{ng} / \mathrm{L}$ or any concentration > $65 \mathrm{ng} / \mathrm{L}^{[8]}$. An acute rise of 10 to $20 \mathrm{ng} / \mathrm{L}$ meets the criteria for "myocardial injury" and may reach a level > $1000 \mathrm{ng} / \mathrm{L}$ during STEMI. One-third of patients undergoing non-cardiac surgery may have an increased preoperative troponin level, and $90 \%$ of MINS occurs in the first two post-operative days ${ }^{[19]}$. Some noncardiac disease processes, including severe chronic obstructive pulmonary disease and end-stage renal disease, can lead to chronically elevated hs-c $\operatorname{Tn}^{[6]}$.

While hs-cTnI is more associated with male sex, age, body mass index, and systolic blood pressure, hs-cTnT is more associated with diabetes and chronic kidney disease. Compared to standard troponin (fourth generation) assays, hs-cTn (5th generation) assays have a higher negative predictive value, leading to earlier detection of AMI by reducing the "troponin-blind" interval, increasing the detection of type 1 MI with a reciprocal decrease in the diagnosis of unstable angina, and are associated with a two-times greater detection of type $2 \mathrm{MI}^{[20]}$ (as shown in Figure 3, the "troponin blind" interval marked as "? Abnormal" in the first panel). Type $1 \mathrm{MI}$ is defined as necrosis caused by coronary atherosclerotic plaque instability, whereas type II MI is necrosis caused by an imbalance between myocardial oxygen supply and demand ${ }^{[20]}$. Elevations of hs-cTn greater than $5 \times$ the upper reference limit have a high (>90\%) positive predictive value for type 1 


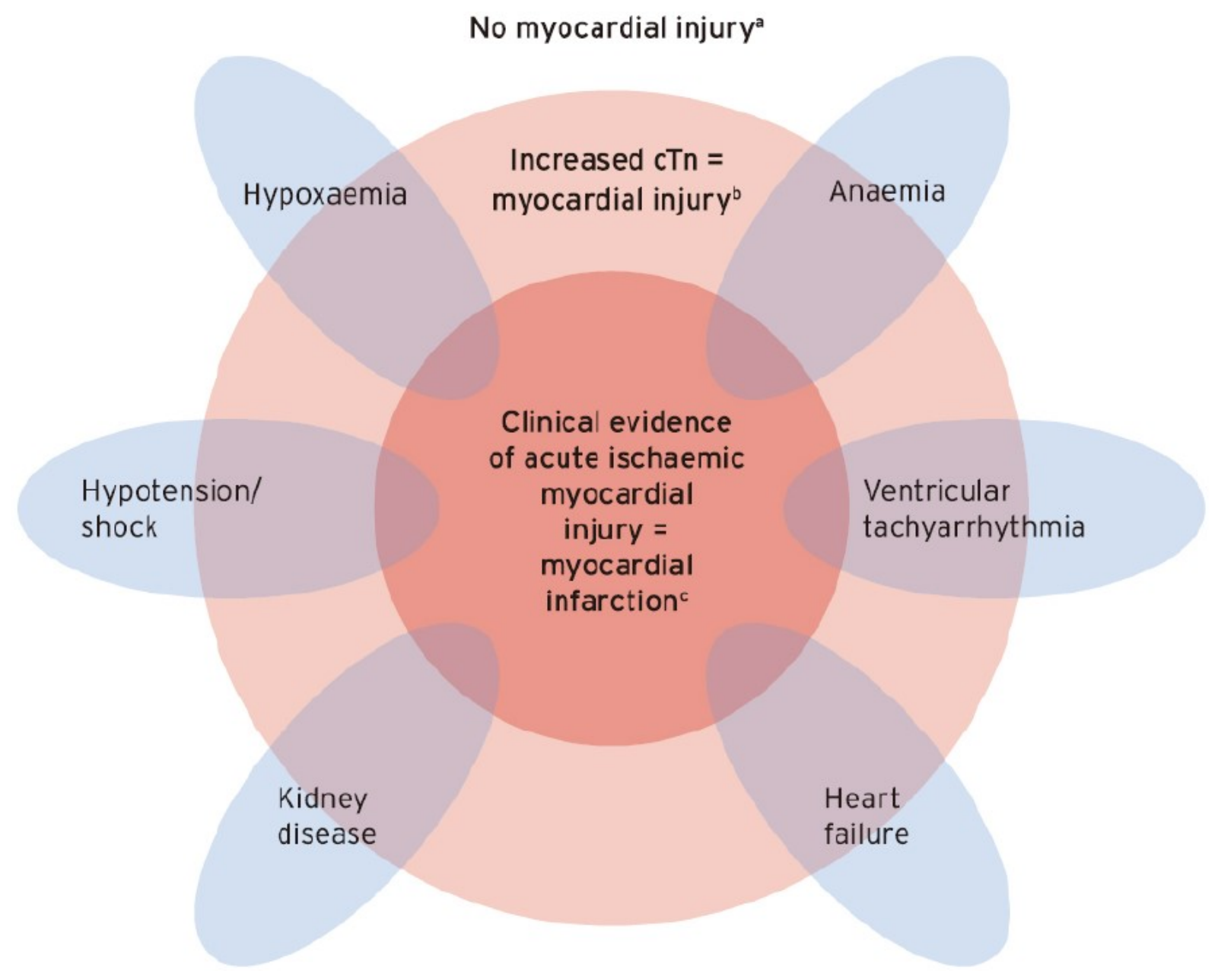

Figure 2. Spectrum of myocardial insult: myocardial injury to myocardial infarction (reproduced with permission) ${ }^{[6]}$. ${ }^{\mathrm{a}}$ No myocardial injury $=\mathrm{cTn}$ values $\leq 99$ th percentile URL or not detectable; ${ }^{\mathrm{b}}$ Myocardial injury $=\mathrm{cTn}$ values $>99$ th percentile URL. $\mathrm{CMyocardial}$ infarction = clinical evidence of myocardial ischaemia and a rise and/or fall of cTn values $>99$ th percentile URL.

AMI, while elevations up to $3 \times$ the upper reference limit have only limited (50\%-60\%) positive predictive value for AMI and may be associated with a broad spectrum of conditions. A rise and/or fall in cardiac troponin levels differentiate acute injury from chronic damage to cardiomyocytes, with a more remarkable change suggesting a higher likelihood of AMI20. Hs-cTn is highly specific for myocardial injury but can also be released in detectable amounts by myocardial stress from extra-cardiac events [Figure 2, Table 1] ${ }^{[21]}$.

The inclusion of "myocardial injury" in the Fourth Universal Definition of MI certainly marks progress, especially with respect to identifying MINS in the perioperative setting. Further work is necessary to understand how myocardial injury relates to true ACS, as this will be essential in developing treatment pathways for this patient population.

\section{Revascularization strategies}

Revascularization strategies for myocardial infarction have varied. For a patient presenting with cardiogenic shock after MI, revascularization of the culprit lesion is favored ${ }^{[2]}$, while for those presenting with STEMI but not in cardiogenic shock, fractional flow reserve - guided multivessel revascularization is favored ${ }^{[23]}$. For NSTEMI, single-stage multivessel revascularization provided superior outcomes ${ }^{[24]}$. As aforementioned, 

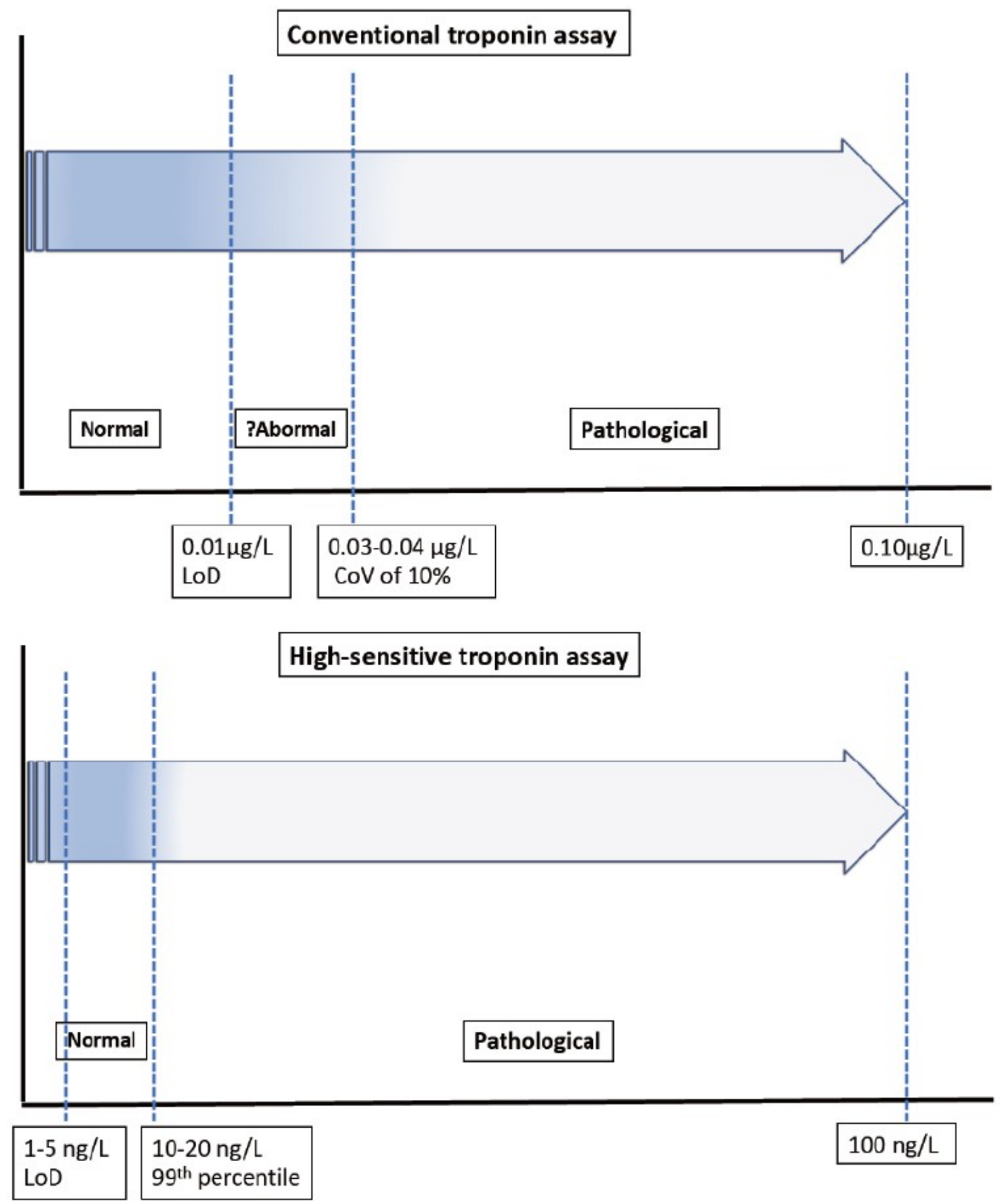

Figure 3. Comparative representation of conventional versus high-sensitive troponin20. LoD: Limit of detection; CoV: coefficient of variation. Note: values for conventional troponin are reported in micrograms per liter while high-sensitive troponin are reported in nanogram per liter.

there is no consensus therapeutic strategy for myocardial injury (or MINS) per $s e^{[6]}$. 
The CARP randomized controlled trial on patients undergoing elective vascular surgeries compared preoperative coronary revascularization (either percutaneous or surgical) to medical management ${ }^{[25]}$. At 30 -day post-operatively, elevations in troponin occurred in $12 \%$ of revascularized patients and $14 \%$ of nonrevascularized patients $(P=0.37)$. After a median of 2.7 years, there was $22 \%$ mortality in the revascularized group and $23 \%$ in the non-revascularized group (RR: $0.98 ; 95 \% \mathrm{CI}$ : $0.70-1.37 ; P=0.92$ ). The median time from coronary revascularization to vascular surgery was 54 days in the revascularization group and 18 days in the non-revascularization group $(P<0.001)$. This was one of the most robust trials showing no reduction in perioperative $\mathrm{MI}$ with coronary revascularization prior to non-cardiac surgery.

Targeting the demand-supply ratio during the perioperative period, the POISE trial confirmed perioperative initiation of beta-blockers reduced the incidence of nonfatal MI but increased the risk of death, stroke, bradycardia, and hypotension ${ }^{[26]}$. In the POISE-2 trial, clonidine failed to demonstrate any benefit ${ }^{[27]}$. The POISE-2 trial also failed to demonstrate a reduced risk of perioperative MI with aspirin but found harm with increased perioperative bleeding ${ }^{[28]}$. Current guidelines suggest continuing the usual dose of betablockers (but not initiating beta blockers immediately before surgery) and re-starting aspirin as soon as possible after surgery ${ }^{[29]}$.

\section{THE KNOWN UNKNOWN}

\section{Risk-modification}

Myocardial damage may be viewed as a spectrum ranging from myocardial infarction caused by coronary occlusion (STEMI) to myocardial injury (mild: "myocardial injury" and severe: NSTEMI) ${ }^{[21]}$. It is unclear, however, how much overlap exists between the latter two groups. In other words, to what extent do patients with prior partial coronary occlusion run the risk of having a complete occlusion when faced with extracoronary (supply vs. demand) mismatch. Also, it is unclear when a mild supply-demand mismatch may convert to a more severe form like NSTEMI. Multiple hypotheses exist but remain theoretical as from the clinical trials mentioned in the above section. It is clear that preemptive revascularization may not prevent MI, while excess or extra perioperative beta-blocker and aspirin may cause more unintended harm than benefit.

\section{Preoperative elevated hsT for screening}

It remains unclear whether we should screen patients for hs-cTn prior to surgery, and when we identify hscTn elevations preoperatively, what it means, and what we should do about them. Sessler and Devereaux ${ }^{[30]}$ have argued in favor of screening all patients for non-cardiac surgery $>45$ years of age; however, we have argued against this, considering medical overtreatment and futility, and the lack of consensus regarding management ${ }^{[31]}$. Recently a small-scale study in elective vascular surgery involving screening 164 patients demonstrated 14 patients had a baseline pre-operative elevated troponin $>14 \mathrm{ng} / \mathrm{L}$, of which 10 underwent coronary angiography with four patients needing coronary stent placement and one patient undergoing coronary artery bypass grafting ${ }^{[32]}$. Despite its limitations, this study provides evidence that screening may identify those patients most at risk of a Type 1 MI (STEMI) from exacerbation of risk factors for Type 2 MI (NSTEMI).

\section{Differences in guidelines}

The 2014 European Society of Cardiology/European Society of Anesthesiology (ESC/ESA) $)^{[33]}, 2014$ American College of Cardiology/American Heart Association (ACC/AHA) (34] $^{[34}$, and 2017 Canadian Cardiovascular Society (CCS $)^{[35]}$ guidelines differ in the recommendations and classification for MINS. The CCS recommends that daily post-operative troponin be measured for 48 to $72 \mathrm{~h}$ for high-risk patients based on cardiac risk indices and BNP or pro-BNP (Class I; Level of evidence B). However, the CCS fails to further risk-stratify or identify strategies to reduce the risk of MINS. 
The ACC/AHA and ESC/ESA recognize MINS but emphasize the paucity of evidence regarding the prevention or management of MINS (Class IIa; Level of evidence C). Suggestions to judicious pre-operative optimization and risk-stratification with a further investigation like stress-echocardiogram or even revascularizations in high-risk patients have been alluded to.

Clinically, for detection of acute MI, multiple "rule-out" and "rule-in" accelerated diagnostic pathways have been devised based on hs-cTn at baseline, at $1 \mathrm{~h}$, at $2 \mathrm{~h}$, and at $3 \mathrm{~h}^{[20,36]}$. Unfortunately, these are accepted but proprietary based, thus lacking universal standardization [Table 2$]^{[37]}$. Also, it is generally accepted that there are four confounders that can influence the level of hs-cTn: age, renal dysfunction, time since onset of chest pain, and sex. In the context of differentiating MINS from acute MI, it is worthwhile for the perioperative physician to be aware of these intricacies.

\section{Management}

Beta-blockers have a theoretical advantage in reducing demand and supply mismatch and hence prevent myocardial ischemia. However, the risk of hypotension leading to other major organ damage needs to be balanced judiciously ${ }^{[38]}$. The CCS guidelines do suggest aspirin and statin (Class I; Level B) that have been found to improve outcomes in myocardial injury patients ${ }^{[35]}$. Dabigatran was tried in a recent trial (MANAGE) to evaluate whether the oral anticoagulant could reduce major vascular complications (nonfatal events: myocardial infarction, non-hemorrhagic stroke, peripheral arterial thrombosis, amputation, and symptomatic venous thromboembolism) in patients suffering MINS after non-cardiac surgery ${ }^{[39]}$. The study did not meet its intended recruitment number due to loss of funding and early termination, the analysis of 1754 enrolled patients, showed no difference in bleeding complications between the dabigatran group and the placebo group, and patients in the dabigatran arm had fewer major vascular events. Composite mortality in dabigatran versus placebo was $11 \%$ vs. $15 \%(P=0.012)$ with vascular mortality $6 \% v$ s. $7 \%$, MI $4 \% v$ s. $5 \%$, and symptomatic venous thromboembolism was $1 \% v s .2 \%$. Of note, the primary safety outcome of composite major bleeding was $3 \% v s .4 \%(P=0.78)$. Technically this trial was flawed as primary outcome was changed mid-way, rates of discontinuation of drug was high $(\sim 40 \%)$, heterogenous patient group and, conceptually, routinely measured troponin in asympotomatic patients without EKG changes remains unclear. In addition to the dabigatran or placebo, aspirin and statins were recommended in the trial patients however there was no standardization of management, a limitation of the study.

\section{THE UNKNOWN UNKNOWN}

\section{Remote ischemic preconditioning}

Myocardial protection by remote ischemic preconditioning (RIPC) of the heart via transient periods of nonfatal periods of ischemia and reperfusion has been studied for decades. However, the translation of this experimental benefit to patients remains controversial. In cardiac surgery patients, while Candilio et al. ${ }^{[0]}$ observed significant benefit by RIPC, a similar benefit could not be replicated in another similar RCT ${ }^{[41]}$. A Cochrane meta-analysis found no positive effect of RIPC on clinical outcomes ${ }^{[42]}$. However, regarding cTn release post-operatively, they did observe that RIPC resulted in measurable cardiac-troponin release at 48 and $72 \mathrm{~h}$. RIPC, despite its encouraging concept and successful pre-clinical studies, has not been able to translate consistently in human trials ${ }^{[43]}$. Over thirty large clinical trials in humans in the past ten years have yielded heterogeneous results. Henceforth, there is still an ongoing debate regarding the benefits of RIPC in preventing myocardial damage, which needs to be addressed with future better designed "precisionmedicine" based trials before universal clinical acceptance. 


\section{Statins}

Preprocedural elevated triglyceride, total cholesterol, and low-density lipoprotein levels have been associated with elevations in post-procedural high sensitive troponin levels ${ }^{[44]}$. A multicenter prospective study looking at MINS and pre-operative statin use demonstrated a significantly lower risk in the statin group (RR: 0.86; 95\%CI: 0.73-0.98; $P=0.02)^{[45]}$. A pilot randomized controlled trial in patients undergoing total hip or knee arthroplasty or repair of hip fractures randomized patients to receive atorvastatin $40 \mathrm{mg}$ daily $v$ s. placebo, starting preoperatively and to continue 45 days after surgery. $80 \%$ patients had detectable hs-cTnI $(>1.1 \mathrm{pg} / \mathrm{mL})$ preoperatively while $20 \%$ patients had a perioperative rise $(\geq 10 \mathrm{pg} / \mathrm{mL})^{[46]}$. There was no significant between-group difference in the change in level from baseline $P>0.10$. The authors concluded that although perioperative MI was common in these three groups of patients, atorvastatin probably did not reduce its incidence, but the study was underpowered to detect differences in MI. A single RCT evaluated whether the continuation of statins perioperatively influenced MACCE on patients with stable coronary artery disease undergoing emergency surgery. The study included 550 patients who were previously taking chronic statin therapy and were randomized to receive rosuvastatin $20 \mathrm{mg}$ or placebo $2 \mathrm{~h}$ before surgery. They found that at 30 days, 10 patients $(3.6 \%)$ in the rosuvastatin group and 22 patients $(8.0 \%)$ in the placebo group suffered a myocardial infarction $(P=0.03)\left[{ }^{47}\right]$. Of note, there was a high use of statins in the screened population, limiting recruitment to the study. A systematic analysis of RCTs evaluated 178 patients who were initiated on a statin prior to vascular surgery. Perioperative statins had no effect on all-cause mortality, cardiac mortality, and nonfatal MI amongst placebo/no treatment and between different doses of $\operatorname{statin}\left[{ }^{48}\right]$. However, the CCS panel believes there is insufficient evidence to make a recommendation on pre-operative initiation.

We hereby reflect the recommendation of CCS that in the presence of plausible data, and since many older adults are already on statins, and low-side effect profile of statins per se, clinically to not discontinue the usual dose of therapy perioperatively.

\section{Platelet reactivity and periprocedural myonecrosis}

In patients having an elective percutaneous coronary intervention, high sensitive troponin was measured, and residual platelet activity was analyzed ${ }^{[49]}$. P2Y12 Reaction Unit (PRU) of over $>208$ was associated with increased hs-Tn release peri-procedurally (OR: 3.39; 95\%CI: 1.87-6.17; $P<0.001$ ). However, PRU of $<200$ has been associated with an increased risk for bleeding. It remains unclear how best to balance the risk versus benefit for P2Y12-inhibitors in the context for MINS, and can P2Y12 platelet-inhibition play a therapeutic or preventive role for the same.

\section{Genomic identifiers for MINS}

Addition of a polygenic risk score (PRS) to the three models for MINS risk prediction: RCRI, a model comprised of entirely pre-operative variables, and a model comprised of combined pre-operative plus intraoperative variables demonstrated that up to $3.6 \%$ of procedures were re-classified ${ }^{[50]}$. Therefore, the authors support the inclusion of PRS in risk-stratification for MINS but refrain against the idea of obtaining genetic information for the sole purpose of risk-stratification as yet.

The prevalence of detectable hs-cTn in the general population has been established. Risk-stratification for cardiovascular events has been suggested utilizing the hs-cTn levels along with cardiovascular risk factors $^{[51]}$. For males and females, respectively, level of hs-cTn $<6 / 4 \mathrm{ng} / \mathrm{L}$ may need lifestyle modification, 6/4-12/10 ng/L may need aggressive lifestyle intervention, and $>12 / 10 \mathrm{ng} / \mathrm{L}$ may need aggressive lifestyle change along with drug therapy. It would be interesting if this could potentially translate into perioperative MINS prevention as well. 


\section{Precision medicine}

The risk of MINS depends on pre-operative risk-factors, intraoperative variables, and genetic susceptibility. In the perioperative period, the interplay of these factors is likely to be dynamic rather than static, given that perioperative interventions are so often aimed to improve the demand and supply ratio, which are subject to change continuously with changes in surgical stimulation, like pain and post-operative mobilization. In the lack of mechanistic etiopathogenesis for MINS currently, along with the pre-operative risk factors, dynamic intraoperative conditions may be integrated by virtue of artificial intelligence (AI) and live automated informatics analysis. For example, the sudden variation of sympathetic responses in relation to surgical stimulus and anesthetic intervention ${ }^{[52,53]}$ may potentially be detected and addressed as it happens for anesthesiology providers to act upon. This may not only help mitigate MINS but also may help identify several sub-phenotypes susceptible to MINS that may go undetected otherwise. Given the advancement in perioperative information technologies, it may not be too difficult to devise a dashboard or parameter for continuous monitoring referred to "MINS view" ${ }^{[12]}$, to help closer dynamic monitoring, dynamic risk mitigation, and even prevent post-operative troponin elevation (MINS). We envision using AI to create a live perioperative "MINS view" that will consider all these factors together ${ }^{[12]}$.

\section{CONCLUSION}

"Myocardial injury" and MINS has been formally recognized as a novel nosologic entity. While diagnosis of myocardial injury has been achieved more consistently with advent of hs-cTn (fifth generation) analysis, however it is yet to be universally standardized and proprietary variations replaced. Formalized clinical management and prevention pathways are yet to be delineated. With increasing test sensitivity to detect cardiac troponins, an elevation of hs-cTn needs to be interpreted as a "continuum" rather than an "absolute" for the detection of the extent of myocardial necrosis. Trending a serial rise or fall should be considered. Clinically, while there exists a recognized void for the treatment of MINS, several strategies directed at prevention and disease-modification of perioperative MINS have been suggested. Strategies like RIPC, even drugs like statins, P2Y12 platelet inhibitors, newer oral anticoagulants like dabigatran have been tested with underpinning scientific principles, but need to be replicated and reproduced before being generalized. Precision-medicine based studies on identifying sub-phenotypes that may be more susceptible to MINS by integrating genomics, metabolomics, patient risk-factors, surgical risk factors, and perioperative hemodynamics has been on the rise. Continuous perioperative dynamic dashboards (like "MINS view") integrating all these factors with principles of deep-learning, aimed at early intervention and active disease modification have been attempted and probably hold a promise in the near future. For now, the major utility and implementation of hs-cTn remains as a "rule-out" pathway for perioperative myocardial infarction which by far remains a more concerning ailment.

\section{DECLARATIONS}

\section{Acknowledgments}

The authors express their heartfelt gratitude for Dr. Mark Nelson and Dr. John Butterworth for their valuable suggestions in preparation of the manuscript.

\section{Authors' contributions}

Made substantial contributions to conception and design of the study and interpretation: Pal N, Worrall E, $\operatorname{Lim} A$

Performed data acquisition, as well as provided administrative, technical, and material support: Pal N, Worrall E 


\section{Availability of data and materials}

Not applicable.

\section{Financial support and sponsorship}

None.

\section{Conflicts of interest}

All authors declared that there are no conflicts of interest.

\section{Ethical approval and consent to participate}

Not applicable.

\section{Consent for publication}

Not applicable.

\section{Copyright}

(c) The Author(s) 2021.

\section{REFERENCES}

1. Weiser TG, Haynes AB, Molina G, et al. Estimate of the global volume of surgery in 2012: an assessment supporting improved health outcomes. Lancet 2015;385:S11. DOI PubMed

2. Weiser TG, Regenbogen SE, Thompson KD, et al. An estimation of the global volume of surgery: a modelling strategy based on available data. Lancet 2008;372:139-44. DOI PubMed

3. Devereaux PJ, Sessler DI. Cardiac Complications in Patients Undergoing Major Noncardiac Surgery. N Engl J Med 2015;373:2258-69. DOI PubMed

4. Smilowitz NR, Gupta N, Ramakrishna H, Guo Y, Berger JS, Bangalore S. Perioperative major adverse cardiovascular and cerebrovascular events associated with noncardiac surgery. JAMA Cardiol 2017;2:181-7. DOI PubMed PMC

5. Botto F, Alonso-Coello P, Chan MT, et al; Vascular events In noncardiac Surgery patIents cOhort evaluatioN (VISION) Writing Group; on behalf of The Vascular events In noncardiac Surgery patIents cOhort evaluatioN (VISION) Investigators. , Appendix 1. The Vascular events In noncardiac Surgery patIents cOhort evaluatioN (VISION) Study Investigators Writing Group., Appendix 2. The Vascular events In noncardiac Surgery patIents cOhort evaluatioN Operations Committee., Vascular events In noncardiac Surgery patIents cOhort evaluatioN VISION Study Investigators. Myocardial injury after noncardiac surgery: a large, international, prospective cohort study establishing diagnostic criteria, characteristics, predictors, and 30-day outcomes. Anesthesiology 2014;120:564-78. DOI PubMed

6. Thygesen K, Alpert JS, Jaffe AS, et al; Executive Group on behalf of the Joint European Society of Cardiology (ESC)/American College of Cardiology (ACC)/American Heart Association (AHA)/World Heart Federation (WHF) Task Force for the Universal Definition of Myocardial Infarction. Fourth Universal Definition of Myocardial Infarction (2018). J Am Coll Cardiol 2018;72:2231-64. DOI PubMed

7. Hedayati T, Yadav N, Khanagavi J. Non-ST-Segment acute coronary syndromes. Cardiol Clin 2018;36:37-52. DOI PubMed

8. Devereaux PJ, Biccard BM, Sigamani A, et al; Writing Committee for the VISION Study Investigators. Association of postoperative high-sensitivity troponin levels with myocardial injury and 30-day mortality among patients undergoing noncardiac surgery. JAMA 2017;317:1642-51. DOI PubMed

9. Beattie WS, Wijeysundera DN, Chan MTV, et al; ANZCA Clinical Trials Network and the ENIGMA-II Investigators. Survival after isolated post-operative troponin elevation. J Am Coll Cardiol 2017;70:907-8. DOI PubMed

10. Sandoval Y, Apple FS, Smith SW. High-sensitivity cardiac troponin assays and unstable angina. Eur Heart J Acute Cardiovasc Care 2018;7:120-8. DOI PubMed

11. Devereaux PJ, Szczeklik W. Myocardial injury after non-cardiac surgery: diagnosis and management. Eur Heart J 2020;41:3083-91. DOI PubMed

12. Colombo R. Myocardial injury after non cardiac surgery: troponin assay is not enough... We need perioperative bearings. Minerva Anestesiol 2018;84:1131-3.

13. Hammarsten O, Mair J, Möckel M, Lindahl B, Jaffe AS. Possible mechanisms behind cardiac troponin elevations. Biomarkers 2018;23:725-34. DOI PubMed

14. Mair J, Lindahl B, Hammarsten O, et al. How is cardiac troponin released from injured myocardium? Eur Heart J Acute Cardiovasc Care 2018;7:553-60. DOI PubMed

15. Marjot J, Kaier TE, Martin ED, et al. Quantifying the release of biomarkers of myocardial necrosis from cardiac myocytes and intact myocardium. Clin Chem 2017;63:990-6. DOI PubMed PMC

16. Smit M, Coetzee AR, Lochner A. The Pathophysiology of myocardial ischemia and perioperative myocardial infarction. $J$ 
Cardiothorac Vasc Anesth 2020;34:2501-12. DOI PubMed

17. Wright DE, Knuesel SJ, Nagarur A, Philpotts LL, Greenwald JL. Examining risk: a systematic review of perioperative cardiac risk prediction indices. Mayo Clin Proc 2019;94:2277-90. DOI PubMed

18. Rodseth RN, Biccard BM, Le Manach Y, et al. The prognostic value of pre-operative and post-operative B-type natriuretic peptides in patients undergoing noncardiac surgery: B-type natriuretic peptide and N-terminal fragment of pro-B-type natriuretic peptide: a systematic review and individual patient data meta-analysis. J Am Coll Cardiol 2014;63:170-80. DOI PubMed

19. Sharma V, Sessler DI, Hausenloy DJ. The role of routine postoperative troponin measurement in the diagnosis and management of myocardial injury after non-cardiac surgery. Anaesthesia 2021;76:11-4. DOI PubMed

20. Collet JP, Thiele H, Barbato E, et al; ESC Scientific Document Group. 2020 ESC Guidelines for the management of acute coronary syndromes in patients presenting without persistent ST-segment elevation. Eur Heart J 2021;42:1289-367. DOI PubMed

21. Nagele P. A simplified proposal to redefine acute myocardial infarction versus acute myocardial injury. Circulation 2020;141:1431-3. DOI PubMed PMC

22. Thiele H, Akin I, Sandri M, et al; CULPRIT-SHOCK Investigators. PCI strategies in patients with acute myocardial infarction and cardiogenic shock. N Engl J Med 2017;377:2419-32. DOI PubMed

23. Engstrøm T, Kelbæk H, Helqvist S, et al. Complete revascularisation versus treatment of the culprit lesion only in patients with STsegment elevation myocardial infarction and multivessel disease (DANAMI-3-PRIMULTI): an open-label, randomised controlled trial. Lancet 2015;386:665-71. DOI PubMed

24. Rathod KS, Koganti S, Jain AK, et al. Complete versus culprit-only lesion intervention in patients with acute coronary syndromes. $J$ Am Coll Cardiol 2018;72:1989-99. DOI PubMed

25. McFalls EO, Ward HB, Moritz TE, et al. Coronary-artery revascularization before elective major vascular surgery. $N$ Engl J Med 2004;351:2795-804. DOI PubMed

26. Wijeysundera DN, Duncan D, Nkonde-Price C, et al; ACC/AHA Task Force Members. Perioperative beta blockade in noncardiac surgery: a systematic review for the 2014 ACC/AHA guideline on perioperative cardiovascular evaluation and management of patients undergoing noncardiac surgery: a report of the American College of Cardiology/American Heart Association Task Force on Practice Guidelines. Circulation 2014;130:2246-64. DOI PubMed

27. Devereaux PJ, Sessler DI, Leslie K, et al; POISE-2 Investigators. Clonidine in patients undergoing noncardiac surgery. $N$ Engl J Med 2014;370:1504-13. DOI PubMed

28. Devereaux PJ, Mrkobrada M, Sessler DI, et al; POISE-2 Investigators. Aspirin in patients undergoing noncardiac surgery. $N$ Engl $J$ Med 2014;370:1494-503. DOI PubMed

29. Levine GN, Bates ER, Bittl JA, et al. 2016 ACC/AHA guideline focused update on duration of dual antiplatelet therapy in patients with coronary artery disease: a report of the American College of Cardiology/American Heart Association task force on clinical practice guidelines: an update of the 2011 ACCF/AHA/SCAI guideline for percutaneous coronary intervention, 2011 ACCF/AHA guideline for coronary artery bypass graft surgery, $2012 \mathrm{ACC} / \mathrm{AHA} / \mathrm{ACP} / \mathrm{AATS} / \mathrm{PCNA} / \mathrm{SCAI} / \mathrm{STS}$ guideline for the diagnosis and management of patients with stable ischemic heart disease, 2013 ACCF/AHA guideline for the management of ST-elevation myocardial infarction, 2014 AHA/ACC guideline for the management of patients with non-ST-elevation acute coronary syndromes, and 2014 ACC/AHA guideline on perioperative cardiovascular evaluation and management of patients undergoing noncardiac surgery. Circulation 2016;134:e123-55. DOI PubMed

30. Sessler DI, Devereaux PJ. Perioperative troponin screening. Anesth Analg 2016;123:359-60. DOI PubMed

31. Pal N, Butterworth J. Troponin is for diagnosis, not screening. Anesth Analg 2017;124:1007. DOI PubMed

32. Mol KHJM, Hoeks SE, van Mieghem NM, et al. Preoperative coronary angiography in vascular surgery patients with asymptomatic elevated high-sensitivity troponin T: a case series. Br J Anaesth 2019;123:565-9. DOI PubMed

33. Kristensen SD, Knuuti J, Saraste A, et al; Authors/Task Force Members. 2014 ESC/ESA Guidelines on non-cardiac surgery: cardiovascular assessment and management: The Joint Task Force on non-cardiac surgery: cardiovascular assessment and management of the European Society of Cardiology (ESC) and the European Society of Anaesthesiology (ESA). Eur Heart J 2014;35:2383-431. DOI PubMed

34. Fleisher LA, Fleischmann KE, Auerbach AD, et al. 2014 ACC/AHA guideline on perioperative cardiovascular evaluation and management of patients undergoing noncardiac surgery: executive summary: a report of the American College of Cardiology/American Heart Association Task Force on Practice Guidelines. Circulation 2014;130:2215-45. DOI PubMed

35. Duceppe E, Parlow J, MacDonald P, et al. Canadian Cardiovascular Society Guidelines on perioperative cardiac risk assessment and management for patients who undergo noncardiac surgery. Can J Cardiol 2017;33:17-32. DOI PubMed

36. Januzzi JL Jr, Mahler SA, Christenson RH, et al. Recommendations for institutions transitioning to high-sensitivity troponin testing: JACC Scientific Expert Panel. J Am Coll Cardiol 2019;73:1059-77. DOI PubMed

37. Sherwood MW, Kristin Newby L. High-sensitivity troponin assays: evidence, indications, and reasonable use. J Am Heart Assoc 2014;3:e000403. DOI PubMed PMC

38. Group PS, Devereaux PJ, Yang H, et al. Effects of extended-release metoprolol succinate in patients undergoing non-cardiac surgery (POISE trial): a randomised controlled trial. Lancet 2008;371:1839-47. DOI PubMed

39. Devereaux PJ, Duceppe E, Guyatt G, et al. Dabigatran in patients with myocardial injury after non-cardiac surgery (MANAGE): an international, randomised, placebo-controlled trial. Lancet 2018;391:2325-34. DOI PubMed

40. Candilio L, Malik A, Ariti C, et al. Effect of remote ischaemic preconditioning on clinical outcomes in patients undergoing cardiac bypass surgery: a randomised controlled clinical trial. Heart 2015;101:185-92. DOI PubMed

41. Hausenloy DJ, Candilio L, Evans R, et al; ERICCA Trial Investigators. Remote ischemic preconditioning and outcomes of cardiac 
surgery. N Engl J Med 2015;373:1408-17. DOI PubMed

42. Benstoem C, Stoppe C, Liakopoulos OJ, et al. Remote ischaemic preconditioning for coronary artery bypass grafting (with or without valve surgery). Cochrane Database Syst Rev 2017;5:CD011719. DOI PubMed PMC

43. Stoppe C, Meybohm P, Benstoem C, Goetzenich A. Remote ischemic preconditioning in cardiac anesthesia: a review focusing on translation. Minerva Anestesiol 2017;83:610-23. DOI PubMed

44. Buturak A, Degirmencioglu A, Erturk M, et al. Impact of increased admission lipid levels on periprocedural myocardial injury following an elective percutaneous coronary intervention. Coron Artery Dis 2015;26:333-40. DOI PubMed

45. Berwanger O, Le Manach Y, Suzumura EA, et al; VISION Investigators. Association between pre-operative statin use and major cardiovascular complications among patients undergoing non-cardiac surgery: the VISION study. Eur Heart J 2016;37:177-85. DOI PubMed PMC

46. Bass AR, Szymonifka JD, Rondina MT, et al. Postoperative myocardial injury and inflammation is not blunted by a trial of atorvastatin in orthopedic surgery patients. HSS J 2018;14:67-76.

47. Xia J, Qu Y, Yin C, Xu D. Preoperative rosuvastatin protects patients with coronary artery disease undergoing noncardiac surgery. Cardiology 2015;131:30-7. DOI PubMed

48. Sanders RD, Nicholson A, Lewis SR, Smith AF, Alderson P. Perioperative statin therapy for improving outcomes during and after noncardiac vascular surgery. Cochrane Database Syst Rev 2013;(7):CD009971. DOI PubMed

49. Choi SY, Kim MH, Hyun KY, Lee MS. Relationship between platelet reactivity and periprocedural myonecrosis in patients undergoing percutaneous coronary intervention. J Invasive Cardiol 2019;31:E369-75. PubMed

50. Douville NJ, Surakka I, Leis A, et al. Use of a polygenic risk score improves prediction of myocardial injury after non-cardiac surgery. Circ Genom Precis Med 2020;13:e02817. DOI PubMed PMC

51. Farmakis D, Mueller C, Apple FS. High-sensitivity cardiac troponin assays for cardiovascular risk stratification in the general population. Eur Heart J 2020;41:4050-6. DOI PubMed

52. Colombo R, Raimondi F, Rech R, et al. Surgical Pleth Index guided analgesia blunts the intraoperative sympathetic response to laparoscopic cholecystectomy. Minerva Anestesiol 2015;81:837-45. PubMed

53. Colombo R, Marchi A, Borghi B, et al. Pulse photoplethysmographic analysis estimates the sympathetic activity directed to heart and vessels. Anesthesiology 2015;123:336-45. DOI PubMed 